\title{
Les actions de masse des années 1920 en Russie : un nouveau spectacle pour la révolution
}

The "mass actions" during the 1920s: a new spectacle for a revolution

\section{Régis Gayraud}

\section{(e) OpenEdition \\ 1 Journals}

\section{Édition électronique}

URL : https://journals.openedition.org/ahrf/12448

DOI : $10.4000 /$ ahrf. 12448

ISSN : 1952-403X

Éditeur :

Armand Colin, Société des études robespierristes

\section{Édition imprimée}

Date de publication : 1 mars 2012

Pagination : 175-193

ISSN : 0003-4436

\section{Référence électronique}

Régis Gayraud, «Les actions de masse des années 1920 en Russie : un nouveau spectacle pour la révolution », Annales historiques de la Révolution française [En ligne], 367 | janvier-mars 2012, mis en ligne le 28 septembre 2012, consulté le 01 juillet 2021. URL : http://journals.openedition.org/ahrf/ 12448 ; DOI : https://doi.org/10.4000/ahrf.12448 


\title{
LES ACTIONS DE MASSE DES ANNÉES 1920 EN RUSSIE : UN NOUVEAU SPECTACLE POUR LA RÉVOLUTION
}

Régis GAYRAUD

\begin{abstract}
Les actions de masse sont des fêtes à thème révolutionnaire apparues en Russie soviétique à partir de 1919. Les plus marquantes célébraient les principaux moments de la Révolution lors de grands rassemblements se tenant le plus souvent dans les lieux mêmes où ces événements avaient eu lieu. Ces spectacles qui permettaient de substituer aux fêtes religieuses des « mystères " révolutionnaires, réunissant des milliers de figurants, fruits d'une collaboration entre l'Armée rouge et des metteurs en scène d'avant-garde, sont caractéristiques d'une époque où l'utopie artistique et l'utopie sociale trouvaient des terrains d'action communs dans le projet révolutionnaire. Dans les premières actions de masse, la population était incitée à participer, une proportion d'improvisation était recherchée. Le cas de La Prise du Palais d'hiver (1920) montre les limites de l'exercice et ouvre la voie à l'utilisation falsificatrice des images qui interviendra peu après.
\end{abstract}

Mots clés : révolution russe, bolcheviks, théâtre populaire, avantgarde, utopie, fêtes révolutionnaire, actions de masse.

On connaît la fascination des bolcheviks pour la Révolution française. Dans cette fascination, la symbolique occupe une place capitale. Les bolcheviks n'avaient pas encore durablement assis leur domination sur l'ensemble de l'ancien Empire russe ravagé par la guerre civile que la mémoire des fêtes révolutionnaires d'après 1789 les incitait à commémorer la fin de l'autocratie impériale et leur propre prise du pouvoir. Si le modèle historique de la commémoration révolutionnaire reste les fêtes républicaines de la Révolution française et particulièrement la plus célèbre 
d'entre elles, la fête de la Fédération du 14 juillet 1790, les réjouissances révolutionnaires qu'a connues la Russie pendant une dizaine d'années à partir de 1919, avec une période particulièrement intense jusqu'en 1924, sous le nom de « commémorations de masse » (massovye prazdnestva) ou d' " actions de masse » (massovye dejstva) - ce dernier terme étant le plus courant - présentent un coloris, une originalité qui les distinguent nettement de leurs devancières mais aussi de nombre de manifestations cérémonielles qui accompagnent ordinairement la célébration des prises de pouvoir. A contrario, à partir de la fin des années vingt, aucune des cérémonies officielles en mémoire des événements révolutionnaires de 1917 ne présentera plus cette originalité. La spécificité proprement théâtrale des commémorations de masse, qui sont autant des spectacles mis en scène avec la collaboration de professionnels du théâtre que des cérémonies mémorielles officielles, caractérise un système limité dans le temps, mais relativement important par son ampleur à l'intérieur de la période considérée. Cet aspect justifie d'ailleurs le terme de "scénisation de masse », qui a également été employé, notamment par le critique Adrian Piotrovski dans ses écrits sur ce phénomène théâtral ${ }^{1}$.

\section{Que sont les « actions de masse »?}

On peut effectivement définir les actions de masse comme des actions de « scénisation » d'une page de l'histoire de la Révolution, réalisées avec la participation d'un nombre important de non professionnels qui tiennent le rôle des protagonistes de l'événement en question, les effets de foule occupant dans ces dispositifs une place prépondérante. Dans sa version idéale, l'action de masse serait un spectacle commémoratif se déroulant en plein air, dans les lieux mêmes où s'est produit l'événement que l'on commémore, dont tous les protagonistes seraient des non professionnels, dont la mise en scène aurait été conçue au sein des cercles culturels ouvriers, où l'intervention des professionnels du théâtre serait $a$ minima et où une large part serait réservée à l'improvisation et à la participation du public. C'est en tout cas l'image qui est immédiatement associée au terme d'action de masse lorsqu' on l'évoque devant le public russe

(1) Adrian Piotrovski (1898-1937), traducteur du théâtre grec, critique, dramaturge, dans les années 1930 directeur artistique des studios de cinématographe Lenfilm, a écrit plus d'une vingtaine d'articles consacrés aux actions de masse dont Пиотровский А. И. Празднества 1920 года, dans Пиотровский А. И. За советский театр! [: Сб. ст.] Л., 1925, с. 9 - 17 et Пиотровский А. И., Гвоздев А.А. Петроградские театры и празднества в эпоху военного коммунизма dans История советского театра. Л., 1933. т. 1. Arrêté pour espionnage, il a été fusillé en 1937. 
d'aujourd'hui. Dans les faits, ce modèle n'a pratiquement jamais été suivi dans tous ses paramètres, et ne s'est développé qu'au cours de l'histoire du genre. Les premières actions de masse, organisées en février-mars 1919 par l'Atelier théâtral de l'Armée Rouge à Pétrograd, eurent d'abord lieu dans un local $\operatorname{clos}^{2}$. Ce fut le cas, le 12 mars 1919, de La Chute de l'autocratie, exécutée dans la salle du Conseil de la Nativité. Mais presque aussitôt, l'intérêt se fit sentir de sortir le spectacle dans la rue. Pour le deuxième anniversaire d'Octobre, le 7 novembre 1919, La Chute de l'autocratie, complétée et remaniée sous le titre de l'Année rouge, est représentée sur la place du Palais - fraîchement renommée place Ouritski, du nom du Président de la Tchéka de Pétrograd qui y avait été assassiné le 30 août 1918 par le socialiste populiste Léonid Kannegiser-, juste en face du Palais d'Hiver, donc là même où s'étaient déroulés les événements de février-mars 1917. C'est, semble-t-il, le premier spectacle à correspondre exactement au modèle du genre. D'autres spectacles du même esprit sont montés, tantôt en salle, tantôt en plein air, au cours de l'année 1919. Ce n'est qu'à partir de l'été 1920 que le genre a achevé de se constituer. On voit alors apparaître des spectacles rassemblant plusieurs milliers de participants dans de vastes espaces ouverts qui permettent l'évolution de groupes aussi nombreux devant un public lui-même important, souvent sur les lieux mêmes des événements reconstitués. Mais dans le même temps, l'aspect improvisé - forcément limité dès l'origine - disparaît totalement; de même, la participation du public se réduit.

En province, toutefois, il semble que l'apport des spectateurs soit resté assez important, plus longtemps que dans les « deux capitales». Les commémorations d'événements locaux sont plus propices à cette intervention du public. En 1923, à Ivanovo, une reconstitution de la grande grève qui y a eu lieu en 1915 met en ébullition la ville toute entière. Les habitants apportent leur contribution à la mise en scène, et de nombreux protagonistes de l'événement originel tiennent à jouer leur propre rôle dans le spectacle. Le « revivre » peut parfois mener à des situations exagérées. À Samarcande, en 1927, pour les dix ans d'Octobre, le public finit par confondre jeu et réalité et se jette sur les acteurs qui jouent les « ennemis du peuple».

(2) Nous empruntons une part de notre documentation aux travaux de Claudine Amiard Chevrel, notamment à son dossier déjà ancien mais fort bien documenté : Claudine AMIARD-CHEVREL, «Les actions de masses à Pétrograd», dans Les Voies de la création théâtrale, vol. VII, Paris, CNRS, 1979, p. 245-275. Voir aussi Émilia Koustova, «Les Fêtes révolutionnaires russes entre 1917 et 1920 : des pratiques multiples et une matrice commune ", dans Cahiers du monde russe, Paris, CNRS, 2006/4 (col. 47), p. 683-714. 
Le recours aux professionnels, notamment pour la mise en scène et les décors, se systématise. C'est le cas déjà pour le spectacle Vers la Commune universelle, interprété, à Pétrograd encore, la nuit du 19 au 20 juillet 1920 pour l'ouverture du $2^{\mathrm{e}}$ Congrès de la $\mathrm{III}^{\mathrm{e}}$ Internationale. $\mathrm{Ce}$ le sera encore plus pour la gigantesque reconstitution de la Prise du palais d'Hiver lors du troisième anniversaire des événements d'Octobre 1917, le 8 novembre 1920. Enfin, à côté des reconstitutions-anniversaires « sur les lieux mêmes », la thématique des actions de masse va évoluer : certaines auront pour sujet un thème plus général de caractère symbolique. C'est le cas déjà de Vers la Commune universelle. On verra bientôt réapparaître à l'ère soviétique un terme oublié depuis des siècles, et inédit en terrain russe - le « mystère » (en russe : misterija) - pour qualifier ce type d'actions de masse (Le mystère du travail libéré, 1920).

Des actions de masse auront lieu dans la plupart des villes russes, mais c'est à Pétrograd, où elles sont nées, qu'elles revêtent l'allure la plus aboutie, les proportions les plus impressionnantes. Des spectacles imposants exigent des lieux grandioses et la ville n'en manque pas. C'est un cliché que de dire que la ville de Pierre et Catherine ressemble à un décor de théâtre, mais il est clair que les concepteurs de ces projets ont exploité le plus possible cet aspect. Bien sûr, les six hectares de la place Ouritski, avec sa forme en arc et son entourage de palais, vont servir pour plusieurs spectacles, dont la Prise du Palais d'hiver. Pour Vers la Commune universelle, la scène gagne le fleuve, qui offre un recul propice aux visions d'ensemble :

« L'action se déroulait sur un espace assez vaste à la pointe est (amont) de l'Île Vassilievski et comprenant : les rampes, l'escalier et le perron de l'ancienne Bourse des valeurs, la place qui s'étend en avant de la Bourse et, dépassant les colonnes rostrales, les passages vers la Néva; les ponts, à droite et à gauche. En outre, des torpilleurs croisant sur le fleuve prolongeaient le lieu théâtral. L'éclairage était assuré par des projecteurs installés à la Forteresse Pierre-et-Paul, sur les ponts, sur les colonnes rostrales et sur les torpilleurs. Les ponts et les passages vers la Néva servaient de lieux d'accès et de dégagement, alors que l'action proprement dite avait lieu sur les rampes et l'escalier de la Bourse et sur l'esplanade devant le bâtiment; c'est-à-dire sur un volume en gradins prolongé au niveau bas par un vaste espace plan largement ouvert vers l'extérieur $»^{3}$.

(3) Claudine Amiard-Chevrel, op. cit., p. 266. 
En 1927, lors des fêtes d'octobre, l'action se déroulait sur la Néva que descendaient et remontaient des navires de guerre, des mannequins gigantesques remplaçaient les acteurs, des effets sonores et musicaux complétaient l'ensemble, permettant au spectacle d'être vu et entendu de loin. Mais l'éloignement du public était manifeste, sa participation était impossible.

L'usage des moyens qu'offre l'Armée rouge n'est pas étonnant. Dès le début de leur histoire, les actions de masse eurent partie liée avec elle. Les responsables politico-culturels de l'Armée rouge, à travers son Atelier théâtral et dramaturgique, furent même à l'origine des premières actions de masse, ce qui s'explique à la fois par l'objectif idéologique suivi, par les conditions historiques et par les nécessités techniques: l'Armée rouge possédait une expérience de l'agit prop grâce aux représentations qu'elle organisait en arrière des fronts de la guerre civile. L'effort de guerre faisait de l'Armée l'institution la plus à même d'organiser des manifestations de cette ampleur : le lien restait fort entre elle et les cercles ouvriers, elle était seule capable d'obtenir l'adhésion d'une masse populaire importante et en même temps la canaliser comme il convenait. Son rôle restera important tout le long de l'histoire des actions de masse, mais il sera capital dans sa première période, celle de la guerre civile : les soldats sont largement utilisés pour figurer les mouvements de foule de l'insurrection, le matériel militaire est mis à contribution. Toutefois, à mesure que les actions de masse essaiment dans les autres villes, et surtout à partir du moment où la période du communisme de guerre laisse place à la NEP, les organisateurs des actions de masse se diversifient, les cercles d'initiative ouvrière, sorte de clubs culturels contrôlés par le Parti, se multiplient dans les entreprises d'État, les organisations de jeunesse peuvent lever à leur tour du personnel et des moyens techniques conséquents. Les lieux des représentations s'en trouvent modifiés. Au cours de la deuxième partie des années vingt, dans les villes secondaires de Russie, les actions de masse ne se limitent plus à la place principale, au champ de foire ou au bord de la rivière, mais gagnent les territoires des usines, et finissent par s'y cantonner. L'Armée n'est cependant jamais très éloignée de ces organisations.

L'ampleur caractérise l'action de masse, qui prend vite des proportions colossales. En transportant leur spectacle de la salle du Conseil paroissial de l'église de la Nativité à l'esplanade du Palais d'hiver, les organisateurs de La Chute de l'autocratie amorçaient cette course vers le gigantisme. Les impératifs de l'agit prop l'exigeaient certainement. On verra plus loin à quelles positions à la fois idéologiques et artistiques 
renvoyaient la présence et plus encore l'implication de la foule. Mais l'élargissement de la scène et l'inflation du nombre de participants ont également pour origine, plus simplement, le succès que rencontrent de telles actions et l'envie d'y participer qu'elles suscitent. Il ne faut bien entendu pas accorder foi au mythe idéologique d'une foule entrant tout entière spontanément dans le jeu et participant pleinement, par sa propre créativité, à la réalisation de ces réjouissances, mais on ne peut pas non plus réduire le phénomène d'expansion de l'action de masse à l'application d'un programme séparé de tout véritable assentiment populaire. Dans le cas des spectacles de l'année 1919 et jusqu'au début de 1920, l'appropriation populaire reste importante, et même fondamentale. Pour une bonne part, les spectacles de cette année sont fondés sur l'utilisation du public comme foule supplétive pour les reconstitutions de manifestations et autres mouvements de figuration, et impliquent une part d'improvisation. Les individus qui composent la foule sont incités, à partir de la trame qui leur est proposée, à prendre la parole, à lancer des slogans, à gérer certains déplacements. L'Année rouge semble, sur cet aspect, avoir constitué une sorte de point culminant.

Une autre caractéristique de l'action de masse en plein air, qui, à notre connaissance, n'a pas fait jusqu'ici l'objet de l'attention qu'elle mérite, est son caractère nocturne. Que ce soient les commémorations pétersbourgeoises de la Révolution de Février ou celles d'Octobre, l'aspect nocturne est à peu près inévitable, étant donné la situation septentrionale de la ville et s'inscrit d'ailleurs dans une reconstitution des événements. Mais l'environnement nocturne favorise une esthétique particulière, que les concepteurs de ces spectacles ne manqueront pas d'utiliser en convoquant feux d'artifice, murs de feux et autres fumigènes favorisant l'image lyrique de l'incendie révolutionnaire. Il est remarquable que tous les spectacles de masse en plein air soient donnés la nuit. Ainsi, pour Vers la Commune universelle, interprétée à Pétrograd le 20 juillet 1920, en raison du long ensoleillement de la ville à cette époque de l'année, les spectateurs sont conviés à partir de 1 heure du matin, afin que le feu d'artifice final puisse avoir lieu après $2 \mathrm{~h}$.

\section{Le cas de La Prise du Palais d'hiver}

À partir de 1920, le gigantisme qui caractérise les actions de masse et finira par avoir raison de cette « spontanéité » initiale, va gravir un palier. Pour La Prise du Palais d'hiver, la plus connue de ces actions, jouée lors du $3^{\mathrm{e}}$ anniversaire d'Octobre, le 8 novembre 1920, le projet initial, décidé 
par la direction politique de la région militaire de Pétrograd, à l'instigation du compositeur de musique Dimitri Tiomkine qui en était membre, envisageait d'utiliser un quartier tout entier et prévoyait la destruction de plusieurs casernes afin de « faire de la place » pour le spectacle. Finalement, à la suite de l'opposition de la Section économique municipale, on s'en tint à la place Ouritski et à une plus sage reconstitution des événements, mais la participation de neuf mille artistes et figurants de toute sorte, ainsi que l'ampleur des moyens matériels utilisés, va fortement marquer les esprits. Tiomkine, pour réaliser cette action de masse, va faire appel à Nikolaï Evréinov ${ }^{4}$, le metteur en scène le plus en vue des dernières années d'avant la Révolution, qui lui-même confiera ses décors au peintre Iouri Annenkov $^{5}$, son décorateur habituel. Pour réaliser ses décors, Annenkov va tirer profit de la configuration générale déjà impressionnante du lieu, en construisant deux estrades le long de l'ellipse que délimitent les deux ailes du bâtiment de l'État-Major de chaque côté de l'arc de triomphe percé en son sein. Ces deux estrades se faisant face ont chacune une longueur de 44 mètres. Celle de gauche supporte des décors rouges figurant les murs de brique des fabriques et des immeubles pour ouvriers, et celle de droite des décors blancs rythmés de colonnes figurant les palais des aristocrates. Chacune est reliée au sol de la place par de vastes escaliers qui permettent les évolutions de la foule, et elles-mêmes sont surmontées d'un praticable supplémentaire qui forme un deuxième étage doublant l'espace de jeu de chaque scène. Dans les «murs de brique » de gauche s'ouvrent de grandes arches dans un décor de murs et de colonnades, figurant comme l'intérieur, vu en coupe, des lieux. À droite, en revanche, les colonnades donnent sur un mur vertical qui restreint la scène et force à un jeu frontal. Reliant les deux scènes, en avant de l'arc de triomphe de l'État-Major, un pont de fausses pierres permet le passage d'un espace à l'autre. Outre ces deux estrades doubles, les escaliers et le pont, le jeu se déroule aussi sur la place que se préparent à investir des unités militaires

(4) Nikolaï Evréinov (1879-1953), dramaturge (comédie Le plus important, 1921) et metteur en scène, était opposé à la conception réaliste de la mise en scène héritée de Stanislavski, partisan du « théâtre en tant que tel » et de la « théâtralisation de la vie ». Il expérimenta un retour aux masques inspirés par la commedia dell arte dans les décors expressionnistes d'Annenkov. Après avoir contribué au renouveau du théâtre russe et à la mise en place du théâtre soviétique, il émigra en 1925 à Paris où il poursuivit son activité de metteur en scène, notamment au cinéma.

(5) Iouri Annenkov (1889-1974), peintre, décorateur de théâtre et de cinéma, et illustrateur russe, fut proche dans sa pratique du cubisme et de l'expressionnisme. On l'a parfois comparé à Georg Grosz. Émigré en France en 1925, il y fit une brillante carrière de décorateur de cinéma. Il est également auteur de souvenirs et d'un roman sur la révolution à Petrograd. 
stationnées dans quatre des cinq rues qui accèdent à la place - artilleurs en fond de scène derrière l'arc de triomphe de l'État-Major, matelots dans le passage qui relie la place à la Perspective Nevski, gardes rouges au débouché de la rue Millionnaïa, régiment Pavlovski du côté de la rivière Moïka - et une compagnie de véhicules automobiles du côté du jardin Alexandre.

L'usage des automobiles est une nouveauté. Ces véhicules surgissent sur la place lors de la scène de la fuite du Gouvernement provisoire. Kérenski, valise en main, se précipite dans un de ces véhicules qui quitte en hâte la place sous les huées au plus fort des « combats ». Peu après, des camions militaires et des blindés apparaissent de dessous le pont central et déversent des artilleurs au milieu de la place, que viennent rejoindre ensuite ceux postés dans les rues avoisinantes. Une batterie d'artillerie est dressée dans le jardin Alexandre. Le spectacle commence par une canonnade. Tout le spectacle est ponctué par des tirs de mitrailleuses et des grondements de canon, les scènes finales formant en ce domaine une apothéose. Au même moment, les soixante-deux fenêtres du Palais d'hiver s'illuminent d'un coup, laissant voir en ombres chinoises les révolutionnaires qui ont investi les étages, retentissent les salves de canons tirées depuis le croiseur Aurore à quai de l'autre côté de la Néva. Des sirènes de bateau se font entendre, le drapeau rouge flotte au fronton du bâtiment. Un feu d'artifice est lancé depuis la place avec pluie d'étoiles rouges, puis l'Internationale se fait entendre. À la fin du troisième couplet, les troupes paradent sur la place, portant des flambeaux.

L'utilisation de la musique dans le spectacle est particulièrement soignée. Dimitri Tiomkine, musicien proche de Prokofiev, qui s'était très tôt spécialisé dans l'accompagnement musical des films muets - et qui fera par la suite, une fois émigré aux États-Unis, et dès l'avènement du parlant, une brillante carrière de compositeur de musique de films à Hollywood, couronnée par quatre Oscars - alterne dans le spectacle une série de musiques frappantes, d'hymnes bien connus censés symboliser les deux blocs opposés avec quelques intermèdes plus légers. Pour cela, il dispose d'un orchestre à la dimension du spectacle : 500 exécutants (!) placés derrière le pont sous l'arc de l'État-Major, recrutés dans les principaux orchestres de la ville. Dans la première scène, par exemple, l'entrée du « révolutionnaire bourgeois » Kérenski est ponctuée par quelques extraits de La Marseillaise, puis une musique comique (la Marche du Crocodile) salue l'arrivée des banquiers et marchands. Arrive la foule des « va t'en guerre », mutilés, invalides traînant leurs béquilles et demandant du sang neuf pour poursuivre la guerre. La Marche du régiment Préobrajenski, 
fière musique militaire du régiment d'élite impérial, forme un contrepoint parodique à ce défilé d'éclopés. Comme au cinéma, la musique joue donc à la fois un rôle d'appui explicatif du jeu de scène et un rôle contradictoire de révélateur ironique lorsqu'il s'agit de montrer l'irréalisme ou le mensonge de ce qui se passe sur la scène blanche. Dans la scène 5 , qui présente une fête sur la scène blanche, l'apparition de blessés en grand nombre au milieu des discours patriotiques y sème le trouble. La Marseillaise qui retentit alors est volontairement jouée fausse.

Tout le long du spectacle, l'opposition entre scènes rouge et blanche est constamment soulignée par une confrontation entre L'Internationale, jouée par l'orchestre, chantée en chœur sur la scène rouge (et, idéalement, par le public) et La Marseillaise, jouée encore dans la scène 7 quand le Gouvernement provisoire dévale les escaliers pour s'enfuir dans les automobiles. La Marseillaise n'est donc plus l'hymne révolutionnaire de 1792, mais un symbole de l'ennemi de classe. Il est remarquable que dans le spectacle Vers la Commune universelle, qui avait eu lieu quatre mois plus tôt - mais qui était, il est vrai, une évocation de la constitution du mouvement ouvrier depuis la Première Internationale - la Marseillaise conservait son identité révolutionnaire. En revanche, l'Internationale tronquée qui retentit à la fin du spectacle, avant la parade finale, n'est pas seulement l'hymne des travailleurs du monde entier. Elle a un statut particulier : c'est en effet dans cette version (traduite par le social-démocrate Arkadi Kotz en 1902), limitée aux trois premiers couplets, que l'Internationale est devenue en janvier 1918 l'hymne national de la RSFSR, puis de l'URSS, et le restera jusqu'en 1944.

De tels spectacles nécessitaient une organisation irréprochable. Pour La Prise du Palais d'hiver, une régie centrale est installée au centre de la place, formant une sorte de mirador à la hauteur du deuxième étage des bâtiments. Onze lignes téléphoniques relient ce poste de commandement à six postes de régie secondaires placés dans chacune des deux estrades et aux quatre accès principaux de la place, à deux batteries de projecteurs placés sur le Palais d'hiver pour éclairer les scènes, au banc des pyrotechniciens, aux canonniers placés dans le jardin Alexandre, à l'artillerie mécanisée qui attend derrière l'arc de triomphe.

Le spectacle de La Prise du Palais d'hiver durait une heure et quart. Outre l'assaut final contre le palais - largement exagéré par rapport à ce qu'il fut en réalité -, il offrait une reconstitution imaginaire de quelques épisodes choisis des derniers moments du Gouvernement provisoire depuis les journées de juillet 1917 et se déroulait d'abord alternativement sur les deux scènes avant de se poursuivre sur la place à partir de 
la débandade du Gouvernement provisoire. Il était divisé en neuf tableaux et ne comprenait pas à proprement parler de dialogues. En revanche, en plus de la musique, les différents éléments de la masse sonore - canon, mitraille, chants, slogans... - permettaient de pallier cette absence. Les pancartes des différents groupes de manifestants et contre-manifestants, que ce soit du côté des révolutionnaires ou des bourgeois, permettaient également de reconstituer pour le spectateur la chaîne narrative. On peut voir là encore une fois, comme pour le fond musical du spectacle, un emprunt aux techniques du cinéma de l'époque, les pancartes tenant le rôle des cartons explicatifs dans le film muet. La scène rouge était destinée à la masse anonyme du peuple, la scène blanche était réservée à ses ennemis. À l'inverse des occupants de la scène rouge, répartis en groupes d'hommes et groupes de femmes de nombre égal, mais guère individualisés, à l'exception des acteurs figurant les bolcheviks de retour d'exil, la scène blanche présentait tout un éventail d'ennemis du peuple bien identifiables : banquiers, marchands, hauts fonctionnaires, généraux, junkers, bataillon féminin, et tout un peuple de bourgeois et de bourgeoises. $\mathrm{Si}$ les figurants de la scène rouge portaient les uniformes de soldats et blouses d'ouvriers et d'ouvrières de tous les jours, et étaient priés de jouer « naturel », les dignitaires, bourgeois et aristocrates de la scène blanche portaient des costumes démesurés, robes chamarrées, chapeaux extravagants rendus grotesques par des ajouts de plumes, costumes surchargés de décorations. De même, les accessoires de la scène blanche portaient tous un caractère exagéré, à l'image des fausses pièces d'or de grande taille qui ruisselaient de coffres grand ouverts, tandis que sur la scène rouge, les accessoires étaient d'authentiques outils et d'authentiques fusils. Mais tous ces personnages étaient des « types » non clairement identifiables.

Un seul protagoniste pouvait être reconnu, et sur la scène, il évoluait d'ailleurs sur un praticable surélevé qui le faisait surmonter tous les autres acteurs, procédé emprunté à La Chute de l'autocratie, spectacle dans lequel, déjà, un personnage était placé sur un piédestal au-dessus des autres protagonistes. Dans la Chute de l'autocratie, ce personnage était l'empereur Nicolas II. Cette fois, il s'agissait d'Alexandre Kérenski, le chef du Gouvernement provisoire. Pour tenir ce rôle ingrat, on avait fait appel à un professionnel, l'acteur de cinéma Pavel Bruk. C'était le seul véritable acteur professionnel et aguerri dans le spectacle. Pour les ouvriers et révolutionnaires, on avait enrôlé d'authentiques ouvriers désignés par les clubs ouvriers de la ville, encadrés par des soldats de l'Armée rouge. En revanche, sur la scène blanche, les personnages étaient joués par des professionnels du cirque et des ballets, et des élèves des écoles 
de théâtre et de danse de la ville. Pour représenter Kérenski, on avait d'ailleurs dans un premier temps pensé également à s'adjoindre les services de vingt-cinq artistes des ballets qui auraient tenu son rôle dans une chorégraphie jouée de façon mécanique. Ce projet, défendu par Annenkov, et Alexandre Kugel, assistant metteur en scène d'Evréinov, fut finalement abandonné de crainte qu'une telle esthétisation et une telle multiplication du personnage de Kérenski finît par troubler le message qu'on souhaitait faire passer : il fallait donner de Kérenski l'image de personnage falot et ridicule que l'historiographie soviétique s'est employée à développer avec succès. Enfin, dans ce spectacle qui procédait par séries d'oppositions franches, le violent contraste entre la masse du peuple, d'un côté, et la solitude de Kérenski, de l'autre, pouvait se justifier autant artistiquement qu'idéologiquement.

Qui étaient les spectateurs de La Prise du Palais d'hiver et où se trouvaient-ils? Occupant le centre de la place, maintenu dans deux espaces rectangulaires délimités par des cordes, le public a été estimé à 60000 personnes debout, qui avaient dû affronter une pluie glaciale en attendant le début du spectacle. Il s'agissait pour l'essentiel d'habitants de Pétrograd et surtout, semble-t-il, de nouveaux habitants, paysans installés depuis peu dans la ville à l'occasion des importants déplacements de population de la guerre civile. La presse de l'époque et les souvenirs d'Evréinov laissent comprendre que les réactions du public furent spontanées, vivantes et même passionnées. Ainsi, quand le spectacle abordait l'échec des journées de Juillet, les spectateurs « revivaient » cet échec et un silence pesant planait sur la place, tandis que la fuite du Gouvernement provisoire avait lieu sous les quolibets. Sur le côté nord de la place, une tribune officielle avec gradins était installée, réservée aux membres de la direction politique de la région militaire, du Soviet de Pétrograd et d'autres invités officiels.

La Prise du Palais d'hiver constituait le clou de tout un ensemble de manifestations qui avaient eu lieu toute la journée à travers la ville. Il en sera de même pour un autre spectacle gigantesque, Le Mystère du travail libéré, réalisé par Kugel. Autre caractéristique commune entre ce dernier spectacle et la Prise du Palais d'hiver, avec cette fois certaines conséquences qui vont dépasser le simple domaine de l'histoire des actions de masse : une équipe de cinéma fut chargée de filmer le spectacle. Le film final, réalisé par le réalisateur Konstantin Derjavine et le cameraman Boris Svetlov (studio Lenfilm, 1920), combine les scènes directement filmées lors de l'action de masse et probablement quelques reconstitutions ultérieures. Une partie des images de ce film, celles où n'apparaissent pas 
les dispositifs scéniques d'Annenkov, notamment celles du Palais d'hiver occupé par la troupe, seront utilisées ultérieurement dans des films de propagande où elles pouvaient passer pour des images tournées lors des événements d'Octobre. Celles où l'on voit la foule se ruer sur les grilles du palais vont être réutilisées dans la version définitive et officielle - dont les images figurant Trotski ont été retranchées - du film Octobre (1927) de Sergueï Eisenstein, lequel est en quelque sorte la première superproduction soviétique et sera tourné avec des moyens qui rappellent ceux des actions de masse : artistes non professionnels, masse immense de dix mille figurants, et même tirs réels en direction du palais. Octobre commence par une déclaration accréditant la vérité historique de tout ce qui se trouve présenté dans le film. Ces images exaltantes mais mensongères d'ouvriers à l'assaut du palais appuieront la mise en place du mythe fondateur le plus important de la mythologie soviétique, celui du soulèvement populaire, de la prise de la Bastille pétersbourgeoise, qui permet de faire d'un coup d'État une révolution.

\section{Les actions de masse au carrefour du théâtre et de la propagande}

Les actions de masse révolutionnaires répondaient à une commande de propagande qui émanait directement des nouvelles autorités, conservant encore à l'époque une certaine autonomie à l'échelon local. Nées à Pétrograd, celles-ci ont eu d'abord comme donneur d'ordre la direction politique de la région militaire. Mais comme on l'a vu avec le cas de Tiomkine, il faut garder à l'esprit l'imbrication qui existait entre un grand nombre d'intellectuels et d'artistes et ces creusets de la propagande qu'étaient ces organismes où l'on cherchait à mettre en place la nouvelle culture populaire du pays. On trouve la même imbrication, dans le domaine des artistes plasticiens, par exemple, pour la mise en place des nouvelles images du nouveau monde en construction. Les peintres et sculpteurs, surtout constructivistes, profiteront quelques années de cette commande de nouvelles formes, à travers la réalisation d'affiches, mais aussi de toutes sortes d'objets où leur art pourra s'appliquer (tissus, papiers peints, décors d'objets de la vie quotidienne). Ces réalisations répondaient pour une part au programme énoncé depuis les années 1910 (notamment dans les milieux futuristes) de « faire sortir l'art dans la rue ». L'art théâtral représentait sans aucun doute pour les donneurs d'ordre de la propagande un médium clef. La propagande offrait aux hommes de théâtre, de leur côte, l'occasion rêvée de pouvoir expérimenter et développer à grande échelle un certain nombre de leurs idées. Dès la décennie 
1910, dans les milieux de l'avant-garde russe, furent organisés des sortes de happenings forcément limités par le nombre de participants, mais qui connurent un assez fort retentissement parmi les intellectuels - mais pas seulement, car les quotidiens en rendaient compte régulièrement. Les vêtements portés par les «futuriens » David Bourliouk, Vassili Kamenski, Vladimir Maïakovski (et sa fameuse blouse jaune, couleur associée à la folie dans la symbolique russe) attiraient la risée du public.

1913, qui fut l'année des grands scandales futuristes, des expositions interdites, des débats publics qui se terminaient en pugilats avec intervention de la police, fut aussi celle où les membres des différents groupes de ce qu'on appelait « l'art de gauche » apparaissaient dans les rues les visages peinturlurés de signes abstraits qu'ils modifiaient plusieurs fois par jour, ces changements étant censés refléter la vitesse avec laquelle le monde moderne multipliait les humeurs de chaque individu dans une seule journée. Surtout, ils proposaient à tout un chacun d'investir ce «nouveau support pour la peinture » que chaque individu portait sur lui. Le manifeste Pourquoi nous nous peinturlurons? signé par le peintre Mikhaïl Larionov et le poète Ilia Zdanévitch en décembre 1913, publié dans le magazine populaire Argus pour inciter la jeunesse à suivre cette nouvelle mode, porte témoignage de cette volonté de sortir l'art hors de ses limites traditionnelles et de le faire partager par tous. Le groupe de Larionov avait compris les limites de la provocation « futurienne» et cherchait, au contraire, à prendre à revers le « bourgeois » en s'appuyant sur une pratique qu'il entendait faire partager à la frange de la population la plus à même de se reconnaître dans ses actions, les lycéens et étudiants à peine plus jeunes que ses membres. La même année 1913, proche de Larionov et les joues peintes lui aussi, le peintre Mikhaïl Le-Dantu organisa une exposition de ses peintures pré-abstraites dans un cinéma populaire de Saint-Pétersbourg. En 1913 toujours, le peintre Kazimir Malévitch et le musicien Mikhaïl Matiouchine réalisèrent une promenade le long de la principale artère de Moscou, portant sur leurs vêtements des objets incongrus - cuillères de bois et légumes. Quand, en janvier 1914, Marinetti, le père du futurisme italien, fit le voyage à Saint-Pétersbourg et Moscou, il fut surpris de constater qu'en Russie, non seulement on suivait son exemple, mais que les slogans extrémistes de Milan étaient dépassés par des jeunes gens qui vivaient désormais « en futuristes $»^{6}$.

(6) Sur tous ces sujets voir Mikhaïl Larionov, Manifestes, traduction et notes Régis Gayraud, Paris, Allia, 1995, et Iliazd, Ledentu le Phare, suivi de Régis GaYraud, Promenade autour de «Ledentu le Phare », Paris, Allia, 1995. 
1913 vit aussi l'apparition d'un théâtre futuriste, avec la pièce de Maïakovski V. V. Maïakovski, tragédie, et surtout avec La Victoire sur le soleil, opéra de Vélémir Khlebnikov et Alexis Kroutchenykh avec musique de Mikhaïl Matiouchine, costumes et décors de Kazimir Malévitch ${ }^{7}$. Costumes «abstraits» (le fameux carré noir de Malévitch $\mathrm{y}$ fait sa première apparition) et partie des dialogues en langage abstrait "d'outre-raison» font de cette œuvre « alogique », équivalent dramatique des peintures de Malévitch du moment, la première pièce d'avantgarde où l'idée de matériau sonore, que l'on retrouvera plus tard dans les actions de masse, s'impose au détriment de l'intrigue et du dialogue traditionnels. Y étaient aussi exploitées en égale mesure les ressources des spectacles populaires, du théâtre forain, du music-hall, du cirque, etc. L'intérêt pour l'art populaire, la peinture d'enseigne et de réclame, les estampes distribuées par les colporteurs, le graffiti urbain, nourrit l'esthétique de tout «l'art de gauche » jusqu'à la veille de la guerre de 1914. La guerre, qui impose couvre-feu et fermeture des lieux de réjouissances, va mettre en sommeil pour quelques années ce mouvement. Il réapparaît presque spontanément dès la révolution de Février puis va disparaître presque totalement, de nouveau, à partir d'Octobre et du début de la Guerre civile, se limitant à quelques poches de résistance. Une partie des artistes d'avant-garde se transporteront dans les pays nouvellement indépendants du Transcaucase, et particulièrement à Tiflis, capitale de la république du Transcaucase, puis de la Géorgie jusqu'au printemps 1921. D'autres, comme Maïakovski ou Malévitch, vont former à Moscou le "Café des Poètes », atelier d'écriture et de propagande actif pour la mise en place de la nouvelle esthétique futuro-constructiviste. Ils vont se rapprocher des groupes anarchistes encore tolérés (le journal Anarxija - « l'Anarchie »-accueille en 1918-1919 les articles théoriques de Malévitch). Le peintre Annenkov, futur décorateur de la Prise du Palais d'hiver, est l'un des décorateurs du café des poètes. C'est sur la base de cette expérience que naîtront au début de 1923, sous l'égide de Maïakovski, le mouvement et la revue Lef (abréviation de Front gauche de l'art), qui développent l'esthétique constructiviste emblématique des années vingt - jusqu'à la disgrâce intervenue en 1926 - en conflit constant avec le mouvement des Artistes et écrivains prolétariens pour dominer la vie artistique en Russie soviétique.

(7) Vélémir Khlebnikov, Alexeï Kroutchenykh, Mikhaïl Matiouchine, Kazimir MaLÉVitch, La Victoire sur le soleil, traduction Jean-Claude Marcadé, Lausanne, L'Âge d'homme, 1976. 
Il est remarquable que, dans l'ébullition de l'année 1913, le monde théâtral professionnel reste en retrait. Les grands metteurs en scène qui s'éloignent du modèle stanislavskien et créeront bientôt le théâtre constructiviste russe sont encore tributaires de l'esthétique symboliste. Vsévolod Meyerhold, Alexandre Taïrov, Evréinov, les trois plus grands metteurs en scène russes de l'époque, ne vont assimiler l'esthétique de l'art de gauche que progressivement, essentiellement à partir de 1918. Les différentes formes de théâtre dit "prolétarien » qui se mettent en place à partir de cette époque, et les actions de masse qui en sont la manifestation la plus remarquable, vont catalyser ce changement de position esthétique, favorisant l'éclosion du nouveau théâtre constructiviste dont on connaît les réalisations ultérieures.

Pourtant, le discours idéologique et artistique qui accompagne les débuts des actions de masse ne s'appuie guère sur le modèle futuriste. Aussi inattendu que cela paraisse, le premier théoricien de ce spectacle populaire de masse est un adversaire déclaré du futurisme, poète adepte du grand mouvement symboliste qui avait dominé la vie artistique du pays depuis la fin du XIX ${ }^{\mathrm{e}}$ siècle et qui servait de repoussoir à tous les autres mouvements nés après lui. Esthète raffiné nourri par l'adoration de l'antiquité gréco-latine, Viacheslav Ivanov $^{8}$ était à la fois un des théoriciens du symbolisme et le tenant d'un retour aux croyances et aux mœurs de l'Antiquité, que sa poésie exprimait dans un décalage constant par rapport au réel, mais qu'il cherchait à reconstituer notamment en rétablissant le culte de Dionysos. Pour ce qui concerne le théâtre, suivant l'exemple de Wagner et de Nietzsche, Ivanov voulait revenir à ses origines grecques. En particulier, il entendait restituer une communion dynamique du peuple dans des actions théâtrales collectives fondées sur le chant choral et la danse. Bien avant la Révolution déjà, il avait théorisé le fait que seul un bouleversement total de la société permettrait d'abattre les cultes usurpateurs, réaliser le nouveau paganisme auquel il aspirait, et reconstruire un peuple d'aèdes et de mystagogues dont le théâtre serait le creuset.

Les symbolistes, et Ivanov au premier chef, non seulement accueillirent, comme la quasi-totalité des artistes de leur temps, avec enthousiasme et soulagement la chute de l'autocratie et l'extase révolutionnaire de 1917, mais participèrent personnellement, pour beaucoup d'entre eux, aux commissions qui se mirent en place pour envisager les nouvelles

(8) Viatcheslav Ivanov (1866-1949), poète, chef de file et théoricien du symbolisme russe, dont la poésie a souvent été accusée de statisme et d'hermétisme. Après avoir défendu dans ses poèmes l'idée du retour à Dionysos, il émigra en 1924 à Rome où il se convertit au catholicisme. 
voies que devait prendre le pays. La prise du pouvoir par les bolcheviks en Octobre 1917 laissa un grand nombre d'entre eux dubitatifs, car ils estimaient que les bolcheviks avaient brisé l'élan de la Révolution et ne pouvaient que chercher à imposer leurs vues. Dans un premier temps, Ivanov n'échappe pas à ce sentiment et sera très sévère, par exemple, pour son camarade en symbolisme, le poète Alexandre Blok, qui adhère pleinement aux orientations prises en octobre et participe aux réunions du Soviet de Pétrograd. Toutefois, sa position change à partir de la fin de 1918, et il entreprend une collaboration qui se prolonge jusqu'en 1920. En 1919, Ivanov dirige le département « Histoire et théorie » de la Section théâtrale du Commissariat du Peuple à l'Instruction publique dont le commissaire est le critique et dramaturge Anatoli Lounatcharski. C'est à ce titre qu'il prononce le 9 mai 1919, devant l'assemblée de la section artistique du premier Congrès panrusse de l'éducation non-scolaire, une conférence qui "fit une énorme impression ». Déclarant qu'à condition sociale nouvelle devait correspondre une nouvelle relation à l'art, et que cette nouvelle relation à l'art entraînerait une éducation populaire supérieure, il proposait d'anéantir les barrières entre création théâtrale et spectateurs. Comme préliminaire, il préconisait la constitution de collectifs théâtraux, de chorales qui interviendraient les jours de fête avec des éléments de chorégraphies, puis l'organisation de fêtes qui favoriseraient l'émergence de nouveaux rituels, fêtes qui se transformeraient ensuite en réjouissances collectives à grande échelle, en plein air, dans des stades et des arènes construits à cet effet, où « tout le privé, le personnel, l'isolé, le souffreteux, le vulgaire - bref, tout ce qu'il y a de petit-bourgeois - tomberait de soi-même et ferait place à la représentation d'actions héroïques de mouvements populaires, et enfin, à l'élément idéal dans les images symboliques d'un mythe, d'un conte, d'une légende $»^{9}$. Le plénum du Congrès reprend et résume ses thèses dans ces termes :

« Le théâtre doit être une collectivité d'actions des masses travailleuses les plus larges. Seule l'action collective dans la création fera du théâtre un facteur véritable de développement harmonieux des membres du collectif communiste [...]. Le théâtre doit progressivement attirer à lui la participation du spectateur au sein des représentations, et c'est ainsi que finira par être créé le théâtre de l'action collective que le prolétariat attend depuis longtemps ».

(9) В. Иванов, K вопросу об организации творческих сил народного коллектива в области художественного действа, dans Русская литература 2-2006, с. 189-198. 
Le succès de l'intervention d'Ivanov fut tel qu'il dut répéter une part de sa communication le 7 août suivant devant le Bureau des communes artistiques ainsi qu'en décembre au Congrès du théâtre ouvrier et paysan ${ }^{10}$.

Les sources intellectuelles d'Ivanov, qui en appelait dans son intervention à Sophocle, Eschyle et Aristophane, ne correspondaient guère aux références habituellement convoquées par les révolutionnaires de 1917. Lounatcharski, le Commissaire du peuple à l'Instruction publique, envisageait avec scepticisme, voire hostilité, le " paganisme » du symboliste, mais ses propres réflexions s'accordaient toutefois au moins sur deux points avec celles d'Ivanov. Lounatcharski avait sa propre théorie du théâtre révolutionnaire qu'il avait mise en forme bien avant la Révolution. Une de ses sources d'inspiration principale était le livre de Romain Roland, Le Théâtre du peuple, dans lequel l'auteur prônait l'émergence d'un théâtre monumental réalisé pour et par le peuple lui-même car « un peuple heureux et libre a besoin de fêtes plus que de théâtres : il sera toujours son plus beau spectacle à lui-même $»^{11}$. Pour Lounatcharski, le théâtre du futur serait un théâtre d'agitation né d'un désir commun, et cette opinion rejoignait Ivanov. L'autre point d'accord était le caractère rituel, religieux du théâtre. "Le théâtre social sera le lieu de mises en scènes collectives de tragédies qui doivent soulever l'âme jusqu'à l'extase religieuse », écrit Lounatcharski en $1908^{12}$. Cet aspect religieux du théâtre révolutionnaire, qui permettrait de communier dans un culte à la Révolution apte à remplacer les anciennes croyances est largement présent dans tout le discours théorique des critiques comme Piotrovski, défenseur tenace du genre, mais également traducteur des classiques grecs et qui partageait avec Ivanov son point de vue sur la refondation collective des mythes et explique aussi la référence aux mystères médiévaux pour définir l'action de masse soviétique. La transe, la démesure des mystères sont invoquées pour justifier le gigantisme des actions de masse.

Le mouvement des actions de masse, dont les origines oscillent entre le symbolisme hellénisant d'Ivanov et le militantisme révolutionnaire de Lounatcharski, le «partage de l'art» futuriste et les nouvelles formes constructivistes, réunit des hommes de théâtre aux conceptions et aux expériences aussi diverses que le chevronné Évréinov, le novice

(10) Sur ce sujet, cf. Роберт Бёрд (Robert BYorD), Вяч. Иванов и массовые празднества ранней советской эпохи, dans Русская литература 2 2006, с. 174-189.

(11) Romain Rolland, Le Théâtre du peuple. Essai d'esthétique d'un nouveau théâtre, $2^{\mathrm{e}}$ édition, Paris, 1913, p. 154 (cité par Claudine Amiard-Chevrel, op. cit., p. 245).

(12) А. ЛуНАЧАРСКИй, Социализм и искусство, dans Книга о новом театре, SPb, 1908, p. 28. 
et futur représentant de l'académisme le plus plat Nikolaï Okhlopkov (auteur d'une action de masse pour le $1^{\text {er }}$ mai 1927 à Irkoutsk), le très classique Kugel, un compositeur sophistiqué comme Tiomkine, des décorateurs modernistes comme Annenkov et plus tard le constructiviste Natan Altman. De cette origine plurielle, il résulte une esthétique particulière, qu'on n'hésitera pas à qualifier de bâtarde, surtout si on la compare aux modèles originaux du symbolisme et du modernisme (futuriste ou constructiviste). Ce qui pourrait le mieux définir l'esthétique des premières actions de masse comme L'Année rouge, Vers la Commune universelle ou La Prise du Palais d'hiver, serait une sorte de binarité simplificatrice poussée à l'extrême des situations, des actions, de la mise en scène. Le principe des deux estrades représentant deux mondes opposés se retrouve dans chacune de ces actions, qui sont pourtant l'œuvre de metteurs en scène différents, aux traditions différentes. Les couleurs utilisées pour les décors, pour les lumières, dénotent également une simplification extrême. Tout comme le poème épique révolutionnaire du symboliste Alexandre Blok, Les Douze, où règnent la neige, la nuit et le feu, les décors d'Annenkov ou d'Altman ne connaissent que ces trois couleurs : le rouge et le blanc des décors, le noir de la nuit. Le «matériel sonore » se limite à l'usage d'hymnes ou de chants révolutionnaires et aux détonations des armes. Cette simplification nous parait le fruit d'un double compromis : entre des écoles artistiques aussi opposées que le symbolisme finissant et le constructivisme en pleine gloire; entre la logique artistique et celle de la propagande. Avec sa symbolique manichéenne et parfois grossière, l'action de masse n'était plus qu'une réinterprétation dénaturée du symbolisme d'autrefois et ne correspondait que superficiellement aux aspirations universelles du constructivisme.

Le projet des actions de masse, partie intégrante d'un plan théâtral prolétarien plus vaste, mais qui en forme la facette la plus brillante et la plus originale, marquera durablement l'inconscient collectif dans la Russie soviétique. Alors même qu'avec les nouvelles dispositions pour encadrer la vie artistique mises en place dès 1926, qui permettront la liquidation des formes artistiques d'avant-garde, le genre s'épuise et finit par disparaître à la fin des années vingt, les parades ou les jeux collectifs très encadrés comme cette partie d'échecs jouée entre deux maîtres soviétiques avec pièces vivantes (pions, cavaliers à cheval, etc.), qui, tel un fantasme de despote oriental, est organisée le 10 juillet 1924 sur la même place Ouritski qui avait vu monter La Prise du Palais d'hiver. Les fêtes de la jeunesse, qui essaiment dans le pays au cours des années 1930, font constamment référence aux actions de masse de l'époque utopique dont elles ne sont plus qu'une forme figée, 
banalisée. Outre le fait que l'expérience ne pouvait pas se poursuivre plus longtemps dans le contexte de la reprise en main de la fin des années 1920, étant donné le danger que pouvaient représenter de telles manifestations, même si la spontanéité initialement prévue en avait été assez rapidement évacuée, le relatif échec de l'action de masse, du point de vue artistique, renvoie à l'échec plus général des mouvements artistiques engagés dans la révolution, et particulièrement de l'avant-garde, sitôt qu'elle a obtenu le statut d'art quasi-officiel. À peine née, l'action de masse n'a jamais pu réaliser totalement l'utopie originelle de l'art réalisé par tous qui occupe l'imaginaire des artistes d'avant-garde depuis les premières années $\mathrm{du} \mathrm{xx}^{\mathrm{e}}$ siècle et l'occupera encore pour plusieurs décennies. Elle a nourri en revanche l'expérience d'autres domaines artistiques, à commencer par le cinéma, dont on a souvent observé la présence non loin des actions de masse mais pourtant parfaitement étanche à la participation du public. On a déjà vu ce que l'art d'Eisenstein, qui exigeait de ses foules de figurants d'organiser elles-mêmes la mise en place de leurs prestations, doit à ces spectacles. On a vu comment la puissance des images d'actions de masse véhiculée par «l'écranisation » (pour reprendre le terme des cinéastes soviétiques) avait pu effectivement contribuer à créer le mythe d'un soulèvement populaire, pierre angulaire d'une croyance quasi-religieuse. C'est bien en cela seulement, peut-être, dans la sinistre réalité de la falsification historique, que le projet mystagogique d'Ivanov a connu quelque réalisation, rejeton tardif et absolument paradoxal d'un symbolisme qui s'était formé dans le refus de toute valeur usuelle, civique et politique de l'art. Enfin, les actions de masse font aujourd'hui partie intégrante des mythologies soviétiques - au sens des Mythologies de Barthes - présentes dans la Russie contemporaine. Elles sont un symbole d'une certaine utopie révolutionnaire qui possède la puissance à la fois attirante et repoussante des mythes. Si les mythes, dont elles devaient permettre l'émergence, ont vite sombré au contact du réel, les actions de masse, aujourd'hui, font mythe elles-mêmes.

Régis GAYRAUD

Professeur de langue et littérature russes Centre de Recherches sur les littératures et la socio-poétique (CELIS) Université Blaise-Pascal (Clermont II) Maison des Sciences de l'Homme 4 , rue Ledru 63000 Clermont-Ferrand Regis.GAYRAUD@univ-bpclermont.fr 
Trois documents sont joints (libres de droits) :

1. La prise du Palais d'hiver, 7 novembre 1920, cadre du film réalisé lors de l'action de masse, avec vue sur la scène rouge.

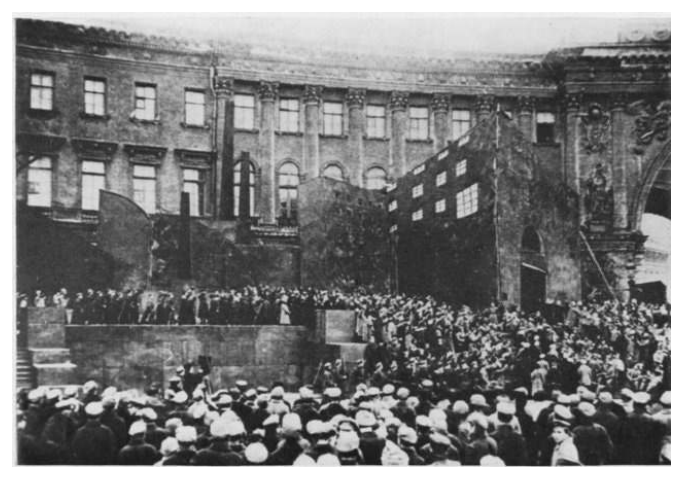

2. Esquisse du décor de Iouri Annenkov pour La Prise du Palais d'hiver (1920), à gauche la scène rouge, à droite, la scène blanche (Musée théâtral Bakhrouchine, Moscou).

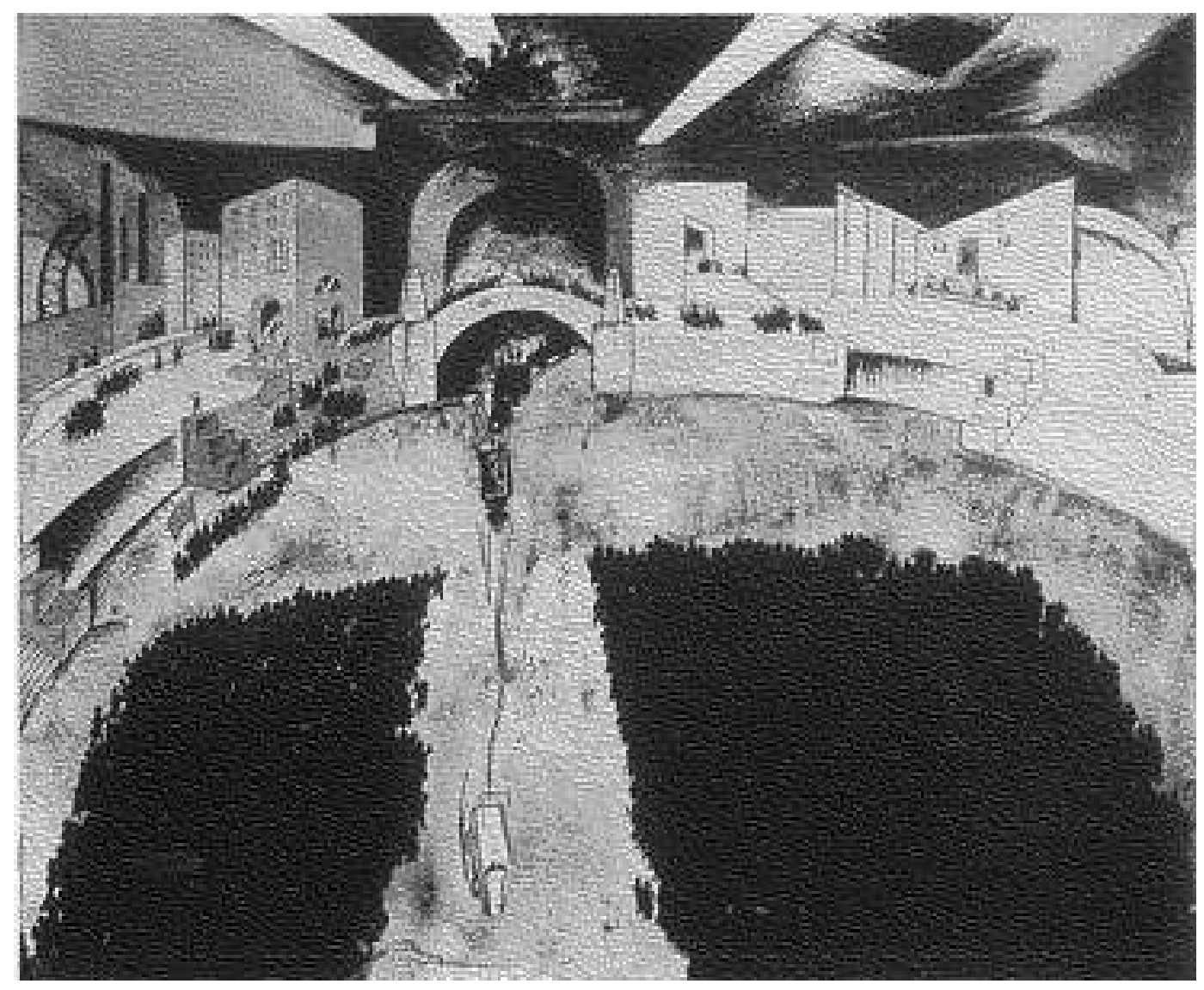




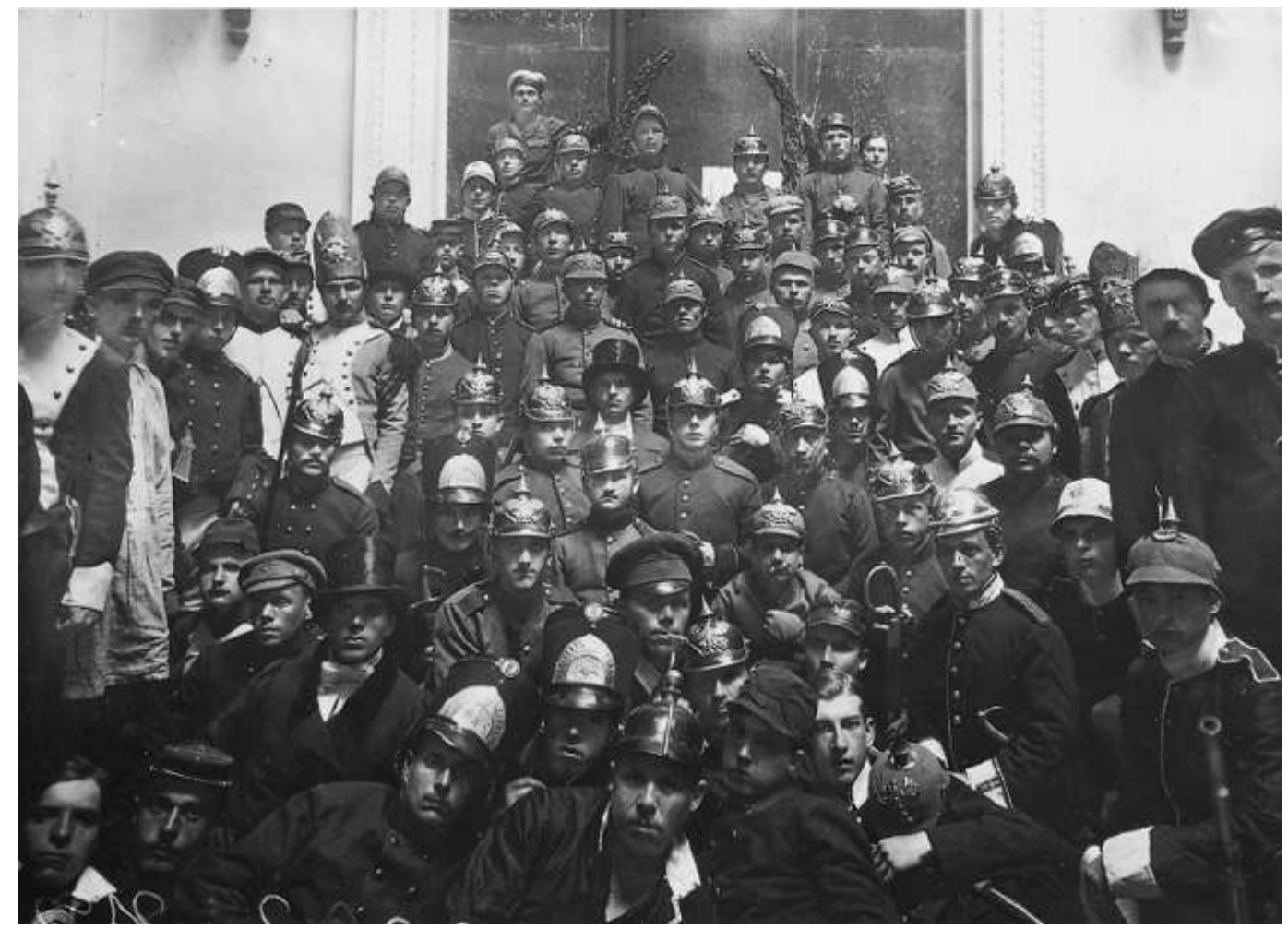

